\title{
The optimization of leased line distribution at the edge of local access network via WAN convergence network
}

\author{
Nor Shahniza Kamal Bashah ${ }^{1}$, Nor Haizon Husin ${ }^{2}$, Syaripah Ruzaini Syed Aris ${ }^{3}$, \\ Norjansalika Janom ${ }^{4}$, Noor Habibah Arshad ${ }^{5}$ \\ ${ }^{1,3,4,5}$ Faculty of Computer and Mathematical Sciences, Universiti Teknologi MARA, Malaysia \\ ${ }^{2}$ Core Network Zone WMSC, TM Putrajaya Exchange, Telekom Malaysia Bhd., Malaysia
}

\begin{tabular}{l} 
Article Info \\
\hline Article history: \\
Received Des 14, 2018 \\
Revised Mar 30, 2019 \\
Accepted Apr 25, 2019 \\
\hline
\end{tabular}

\section{Keywords:}

Convergence network

Leased line

Network resource

Synchronous digital hierarchy

Time slot

\begin{abstract}
Managing the multiple services of the leased line at the same location is quite critical especially when the resource become highly utilized. Bundling the several network resource components into a one box by simplifying the multiple networks to a single network can help to reduce the utilization of network resources. This paper presents a study on optimiziation of leased line distribution at the EDGE of Local Access Network via WAN convergence network. In this study, a WAN Convergence Network is designed which intends to use only a single leased line network in a location rather than multiple leased lines. It is using a simple concept of SDH structured which channelized the time slots and control the transmission line. The time slots will be relocated from the multiple leased lines into a WAN Convergence Network and it will be de-multiplexed through the Data Circuit Terminating Equipment (DCE) at the customer premises. The WAN Convergence Network design starts from the Digital Data Network (DDN) until the DCE which includes the Local Access Network. This approach will be able to save the network resource especially the time slots, cable port, DCE and consequently avoid adding new network infrastructure. This research will result to the new network design which offer multiple leased line networks at the customer end by using only one dedicated leased line network namely WAN Convergence Network.
\end{abstract}

Copyright $\odot 2019$ Institute of Advanced Engineering and Science. All rights reserved.

\section{Corresponding Author: \\ Nor Shahniza Kamal Bashah, \\ Faculty of Computer and Mathematical Sciences, \\ Universiti Teknologi MARA, \\ 40450 Shah Alam, Selangor, Malaysia. \\ Email: shahniza@tmsk.uitm.edu.my}

\section{INTRODUCTION}

Data communications and networking or ICT is the fastest growing technologies and it has become an important element in market business today. The needs of ICT are more critical especially when network provider is unable or delay in delivering the services due to inadequate resources in network infrastructure [1-3]. Therefore, network providers have been concentrating on their network infrastructure and services in order to facilitate and satisfy the customer while enjoying their services. It is also very crucial to ensure that the infrastructure resources are managed effectively. Many research has been conducted [4-7] to determine the best solution in offering continuous services at the customer end. Researchers have been discussing and identifying the issues $[8,9]$ which relates to network performance and the service quality delivered to the customer. Based on the analysis and requirements identified from the literature studies, there is a possibility to bundle the component of network elements into a one box and become an integrated solution.

The proposed solution is called Convergence Network [10-12], which will simplify the basic components such as network element and virtualization by consolidating it into a single network and optimize 
the services to the customer. However, the suggestion need to be examine thoroughly especially in terms of network performance and the quality of services delivered. Thus, this research will study on how the converged network can optimize the leased line services to the customers at the edge of the local access network. It will first review the existing leased line provisioning process in order to identify the problem and what are the important elements that can help to solve the problem.

This paper presents the result on optimization of leased line distribution at the EDGE of Local Access Network via WAN Convergence Network which starts with the explanation on the existing scenario of Leased Line Network Distribution at the Local Access Network. Next, the design and implementation of the WAN Convergence Network using a simple concept of SDH structured which channelized the time slots and control the transmission line is explained. The technique used is by relocating the time slots from the multiple leased lines into a WAN Convergence Network and de-multiplexed through the Data Circuit Terminating Equipment (DCE) at the customer premises. Then, the analysis of the WAN Convergence Network implementation is presented, where it proves that it is able to save the network resource especially the time slots, cable port, DCE and consequently avoid adding new network infrastructure. Finally, the paper ends with conclusion that the network infrastructure can be optimized through minimizing the cost of infrastructures development and operation, reducing the risks of service deployment and reducing the operation time to market for new services.

\section{EXISTING SCENARIO OF LEASED LINE NETWORK DISTRIBUTION AT THE LOCAL ACCESS NETWORK}

Open Systems Interconnection (OSI) layered framework consists of seven separate but related layers, each of which defines a segment of the process of moving information across a network [13]. The layers involved when a message is sent from device A to devices B. Before that, the physical layer usually coordinates the functions required to transmit a bit stream over a physical medium [14]. It deals with the mechanical and electrical specifications of the interfaces and transmission medium. It also defines the procedures and functions that physical devices and interfaces have to perform for transmission to occur. Then, it may pass the message through the intermediate nodes which named as the IPVPN Network. Usually the intermediate nodes contain the first three layers of the OSI model [15]. In developing the model, the designers distilled the process of transmitting data down to its most fundamental elements. Then, the network will identify which networking functions had related uses and then will collect those functions into discrete groups that became the layers. Then the message will be distributed to the receiver end via the dedicated local access network through the respective devices. Local access network usually distributes the message to the receiver end via the assigned dedicated network. Usually, the multiple networks will use separate network resources from the DDN Network to the local access network which consists of the time slot and physical port termination as depicted in Figure 1.

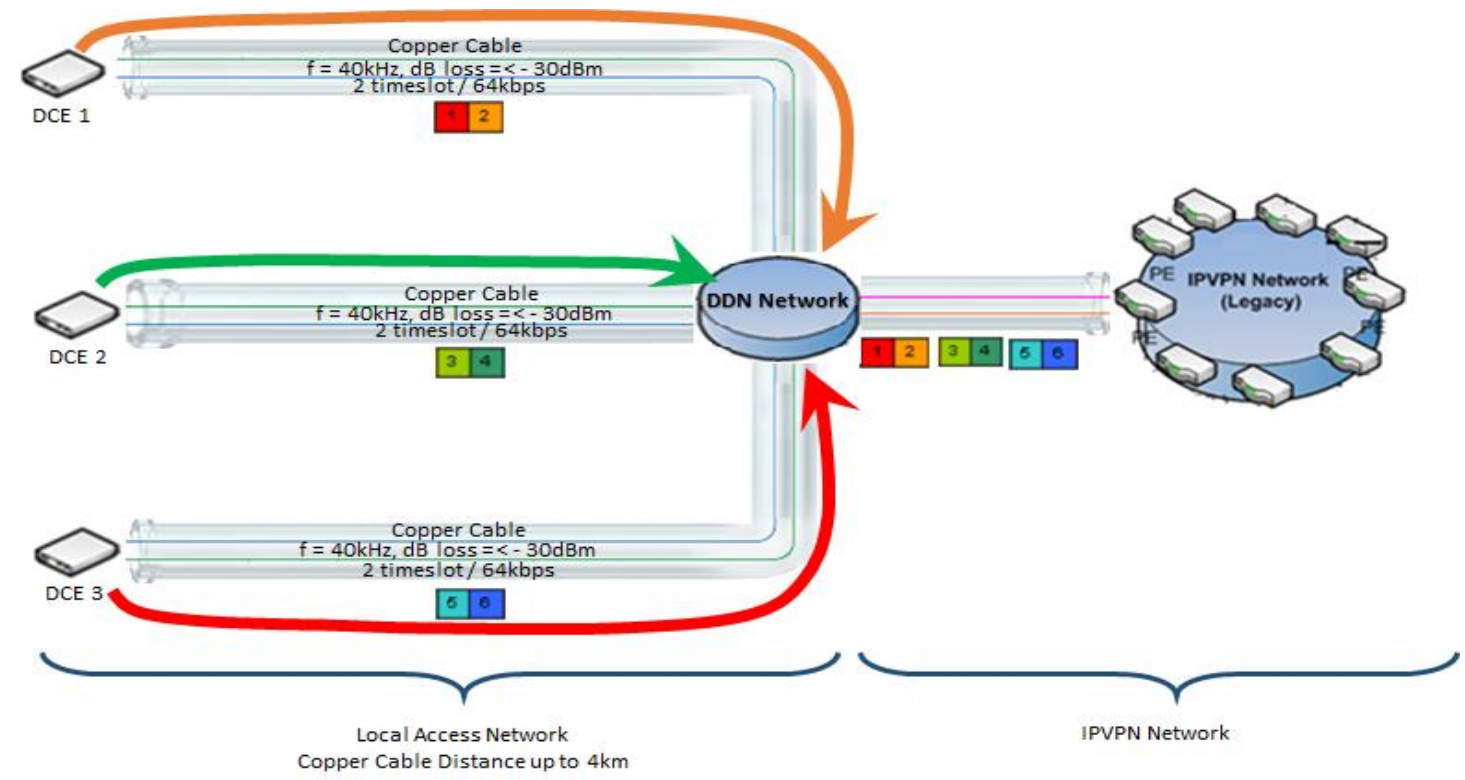

Figure 1. Existing leased line network diagram at the local access network 
It means, each of the networks use separated resources like DDN Network Resources [16] such as time slots, DDN Port and DCE. The Local Access Network resources consist of Cable Port, Internal Cabling and Electrical miscellaneous. The situation will contribute to the high utilization of the resources for the bulk of leased line provisioning. Therefore, the main concern here is how to reduce the network resources and optimized the network output without adding new infrastructure to the network. Thus, this research proposed a process of designing a simplified leased line network architecture which is summarized in Figure 2. From the process, the solution to optimize the network distribution at the edge of the local access network will be discovered. Hence, Figure 1 proves that the time slots is the important element in order to reach the main objective of the research, which is to reduce the resources of the network infrastructure at the local access network $[17,18]$.

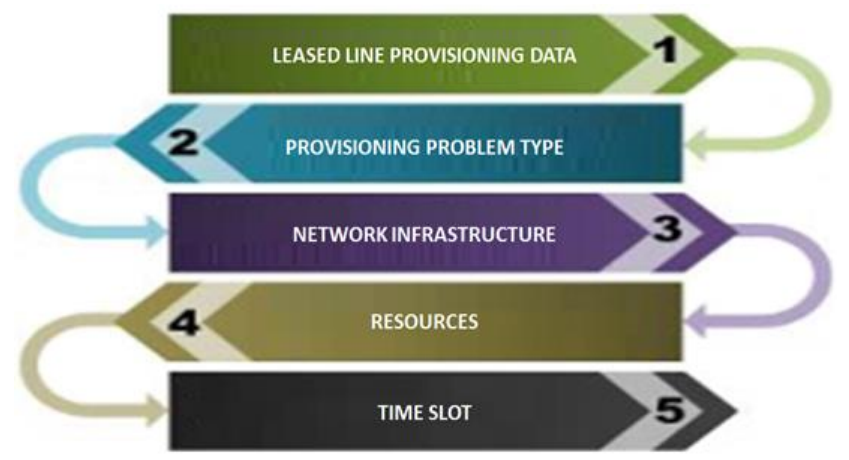

Figure 2. Overview process

Figure 3 is the process on how to provision any new leased line network for the WAN Network. The process indicates that the time slot and port at DDN Node is a critical point where it can push network planner to adding a new infrastructure of network when the capacity meets the limit. In a nutshell, if some innovation to the time slot utilization can be done, it may help to solve the crucial problem. Figure 3 shows that if the time slots are well organized, the leased line provisioning process will be easier and at the same time it can offer optimum distribution at the edge of the local access network without adding new infrastructure to the network $[19,20]$.

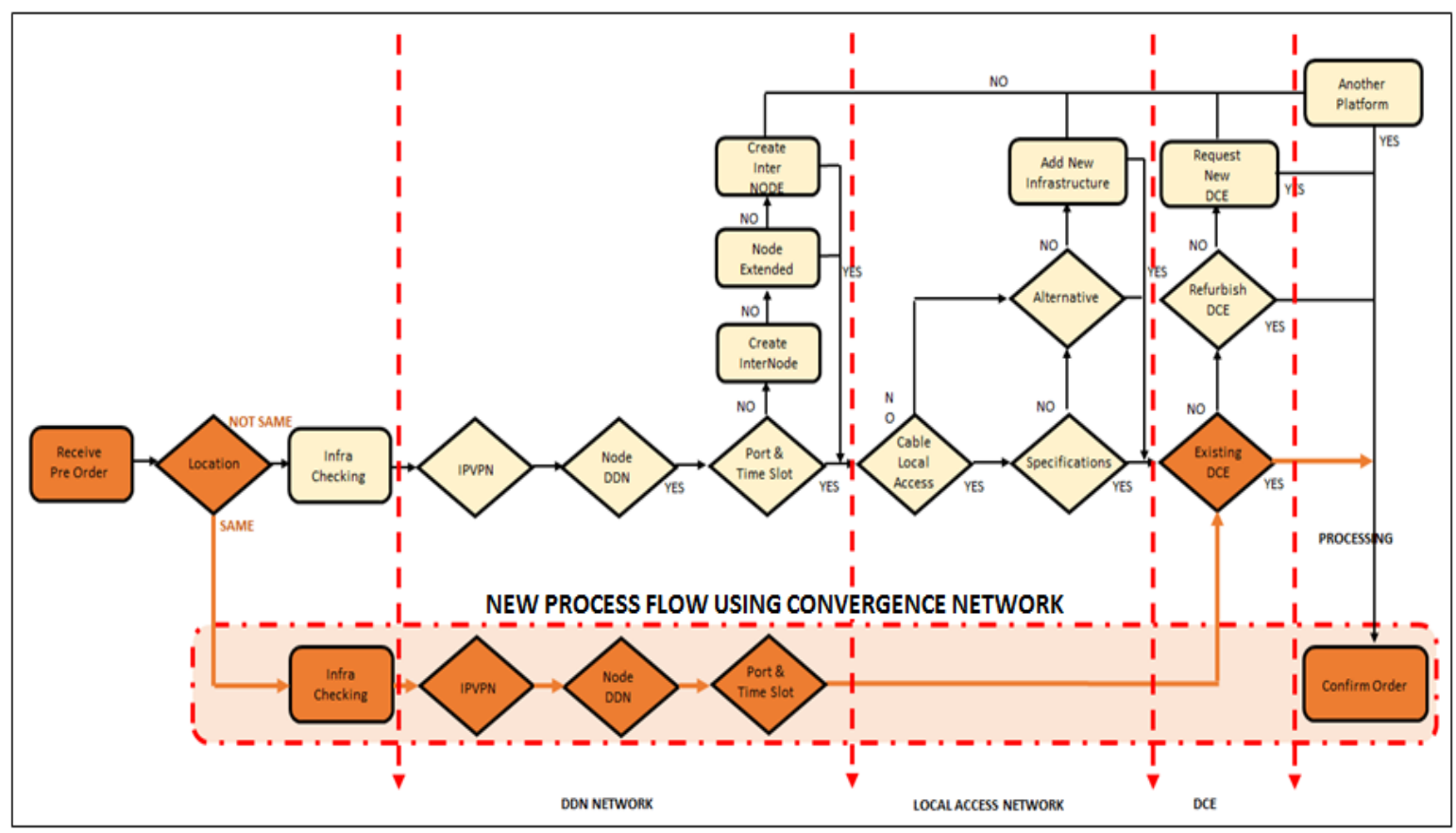

Figure 3. Different provisioning between existing and convergence network 


\section{DESIGN AND IMPLEMENTATION OF WAN CONVERGENCE NETWORK}

WAN Convergence Network is the new network design that is proposed in this research, where it is using only one physical network but contains multiple WAN Networks virtually from DDN Node to the DCE at the receiver end as depicted in Figure 4 [21, 22]. Figure 5 shows the DCE module that need to be added for multiple users at the last end.

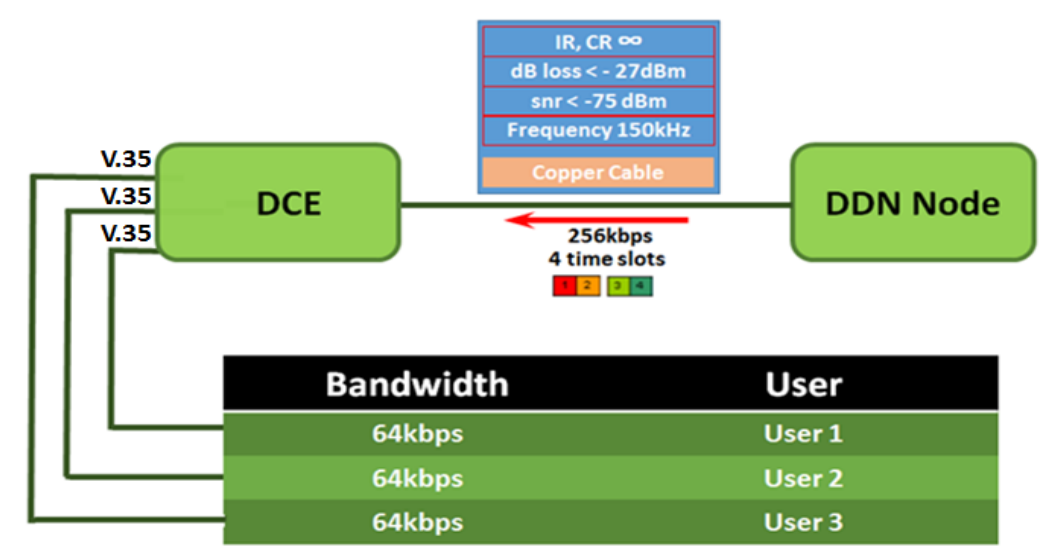

Figure 4. New network design

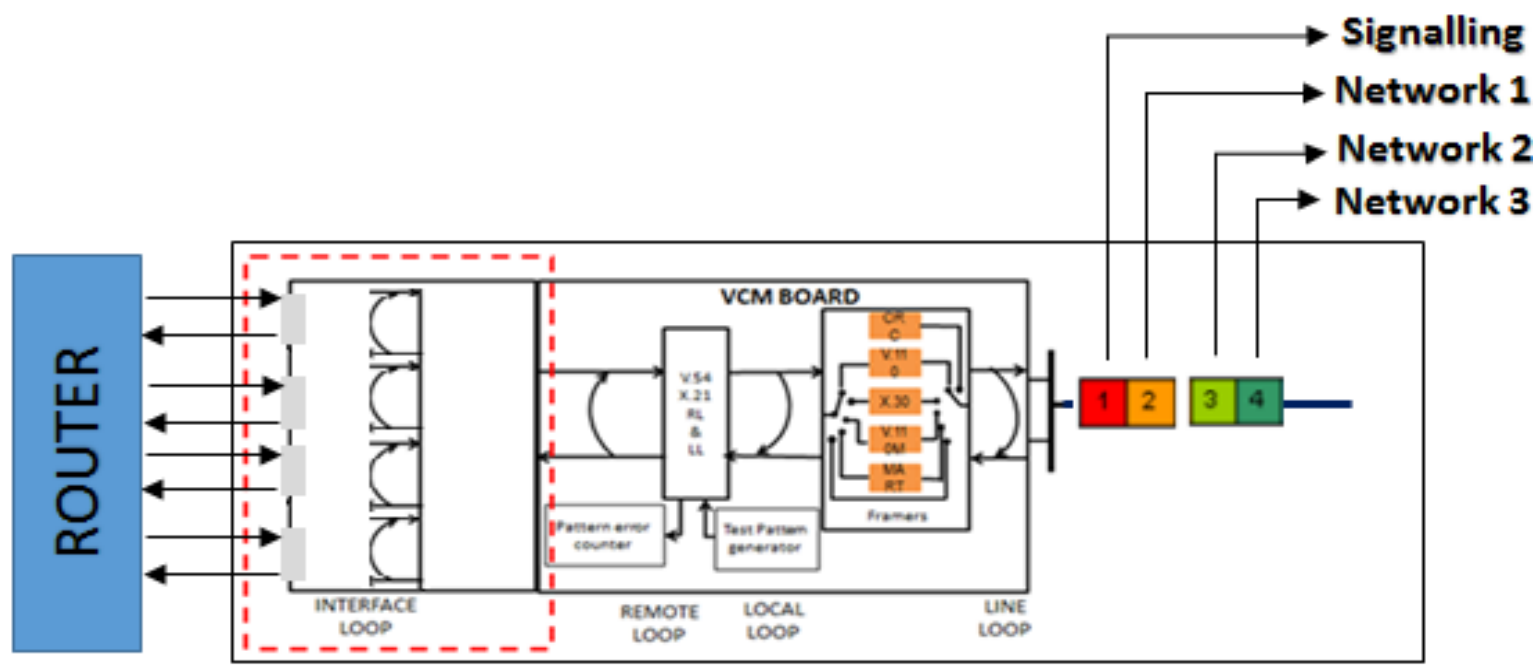

User Access

Figure 5. New module at DCE

Thus, this research proposed two phases to produce the convergence network [23]. Phase 1 determines the function and technical requirements for the transmission medium which fulfilled during the provision of the convergence network. Three parameters used in this phase which are dB loss, frequency test and noise. While Phase 2 relocates time slot and define time slot functions for communication synchronization between the DDN Network and DCE.

In Phase 1, the WAN Convergence Network will be set up which intends to make the multiple networks become only one network. Therefore, the new infrastructure of WAN Convergence Network will be set up physically. However, technical justification for the convergence networks need to be done first because of the existing WAN Network is using low frequency specification $(40 \mathrm{kHz})$ due to only one network covered. The convergence network required high frequency $(150 \mathrm{kHz})$ to deliver the multiple networks.

Phase 2 will relocate the time slot from DCE 1 and DCE 3 to the WAN Convergence Network where the required time slots are depending on the total bandwidth at the receiver end. Then, BERT will be done after 
new DCE for WAN Convergence Network is installed to ensure that the WAN Convergence Network from DDN Network to the DCE 2 which includes HDSL is working without error. Figure 6 shows the steps on how to relocate the time slots.

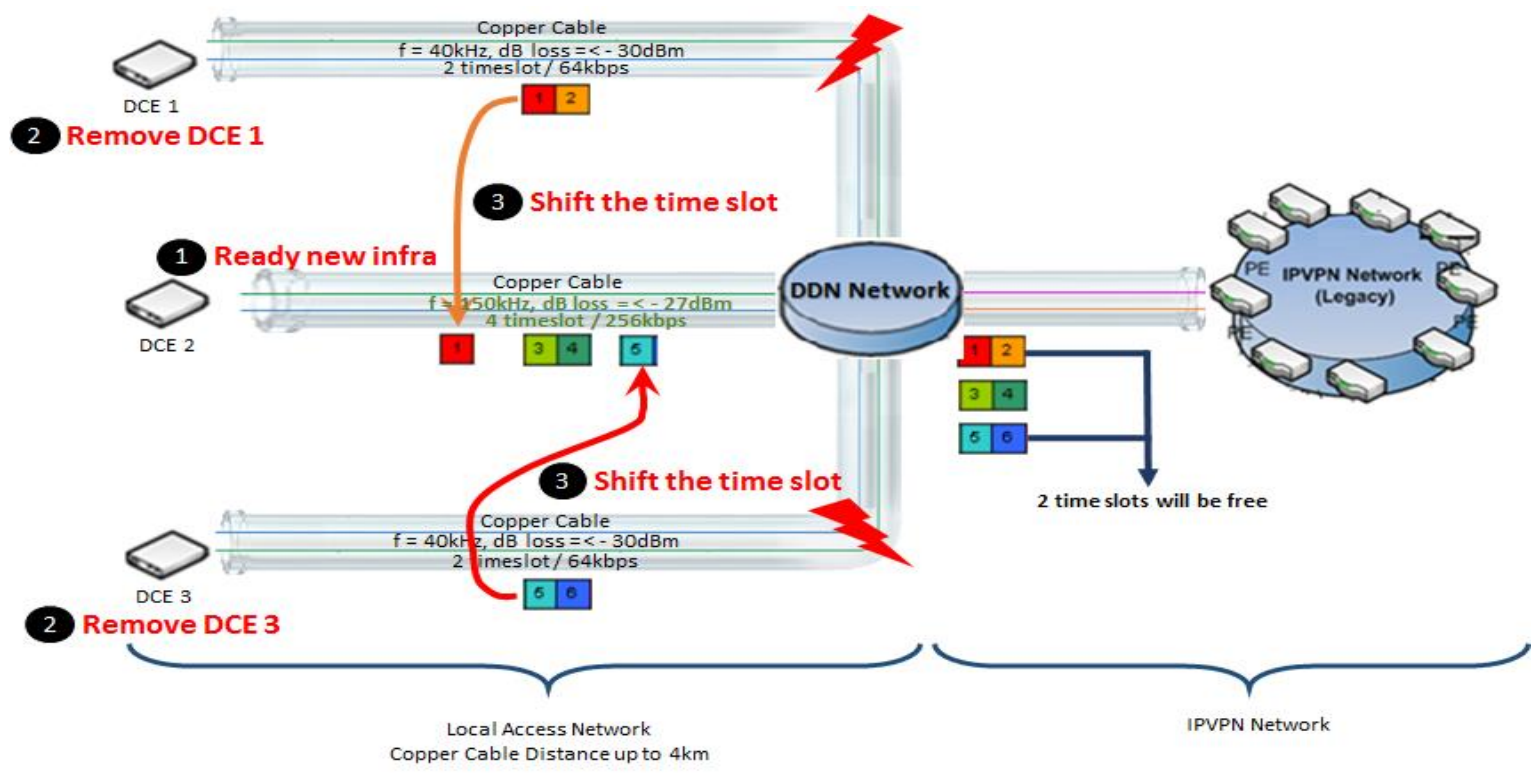

Figure 6. Step to relocate the time slot

Lastly, each of V.35 interface at the DCE will be tested for error checking [24]. Figure 7 shows the WAN Convergence Network which contains the three different networks running virtually through a single network [25].

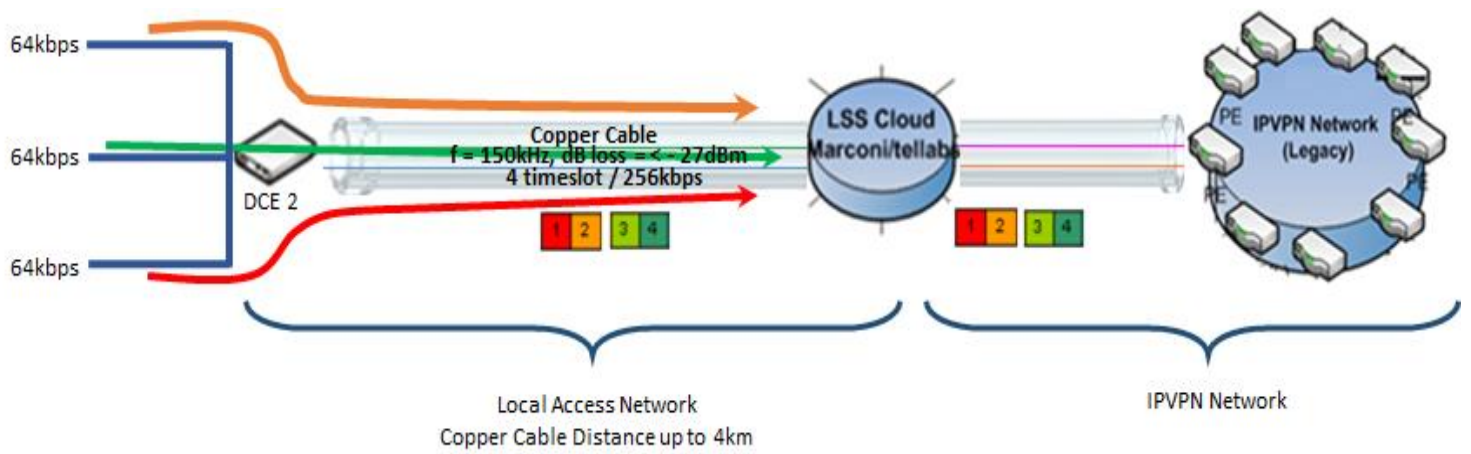

Figure 7. All three networks merge to WAN convergence network

\section{RESULTS AND ANALYSIS}

In this section all the results of WAN Convergence Network implementation are analyzed and thoroughly discuss. The analysis of the results is tabulated in graphs and the discussion will verify, compare and justify the set of results between the existing network and WAN Convergence Network proposed in this research.

\subsection{Analysis for the transmission medium quality between existing network and convergence network}

Figure 8 shows the WAN Convergence is using the high frequency $150 \mathrm{kHz}$ rather than existing network design which is using the low frequency $40 \mathrm{kHz}$. Using the standard recommended reading for the $\mathrm{dB}$ loss and noise level, the WAN Convergence is believed can deliver the multiple network virtually using the

\footnotetext{
The optimization of leased line distribution at the edge of local access ... (Nor Shahniza Kamal Bashah)
} 
same network infrastructure. It is proved by the same pattern as shown by the comparison graphs between the existing network design and WAN Convergence Network.
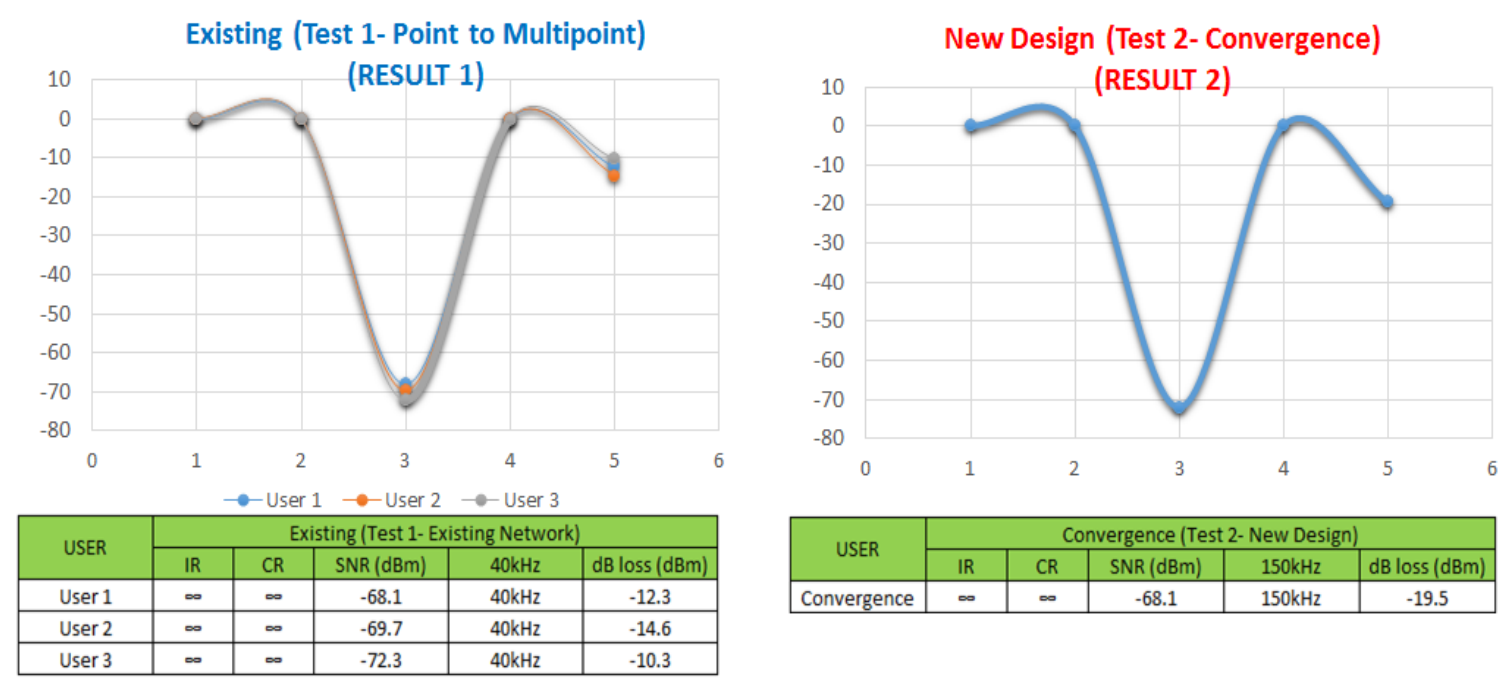

Figure 8. Result of transmission medium quality

\subsection{Analysis for the network connectivity between existing network and HDSL network}

The network connectivity is very important to see the continuity and connectivity of the life network all the time. Any counter of bit error will contribute to the failure of the signal or message sent to the destination. Therefore, the WAN Convergence Network should be error free. From the graph in Figure 9, it shows that the WAN Convergence Network has the same pattern as practiced by the existing network design. Therefore, it can be concluded that the WAN Convergence Network has good network connectivity even though less time slots are used rather than multiple leased line.

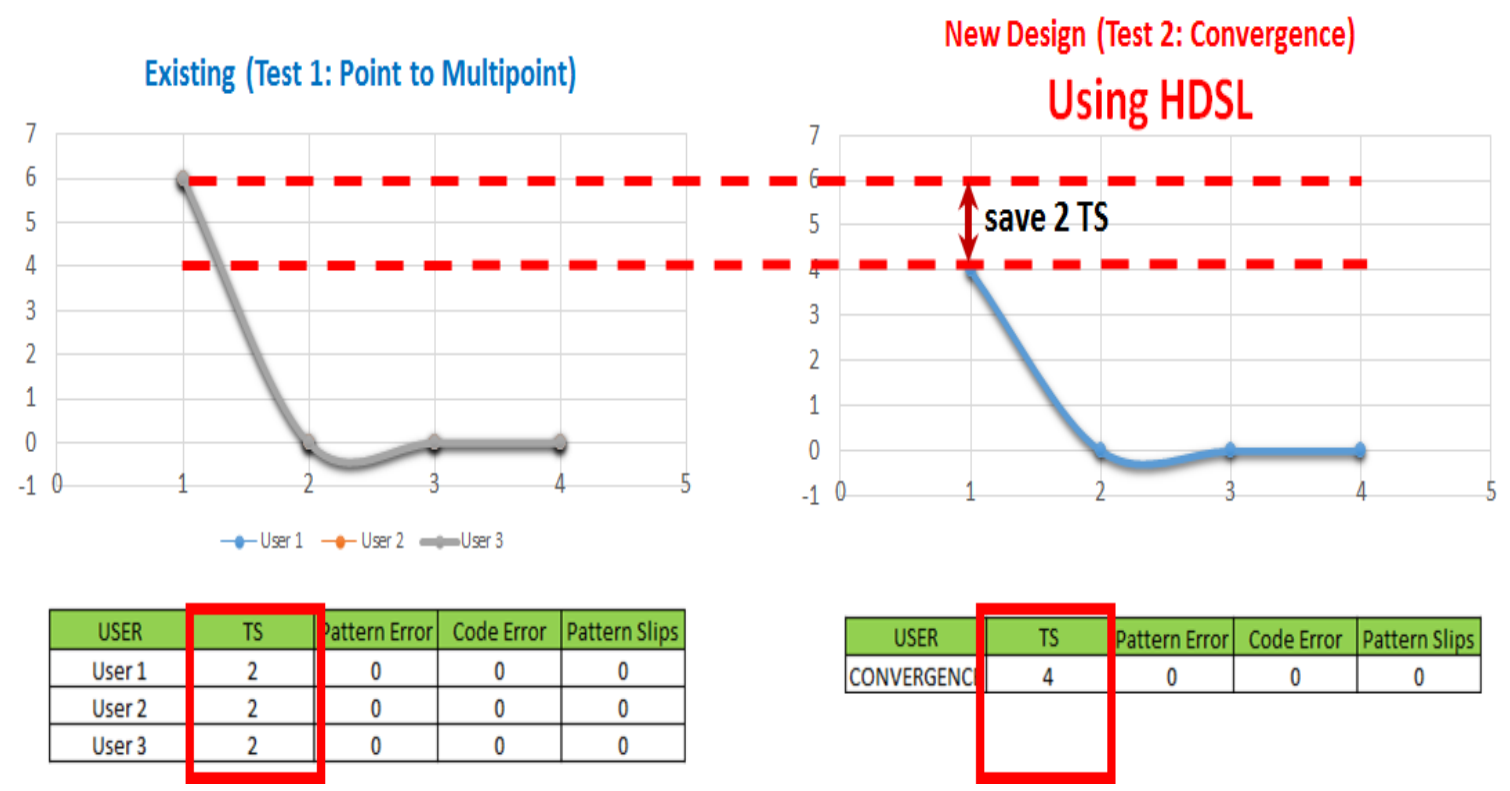

Figure 9. Result of BERT for transmission medium 


\subsection{Analysis for the network connectivity between existing network and convergence network patch to DDN Network}

Once the transmission medium is successfully tested with no error, the network will be extended and patching to the DDN Network. The DDN Network is ready with 4 time slots for bandwidth and synchronization with the DCE at the customer premises. From the BERT test it is indicated that WAN Convergence Network is error free as shown in Figure 10.

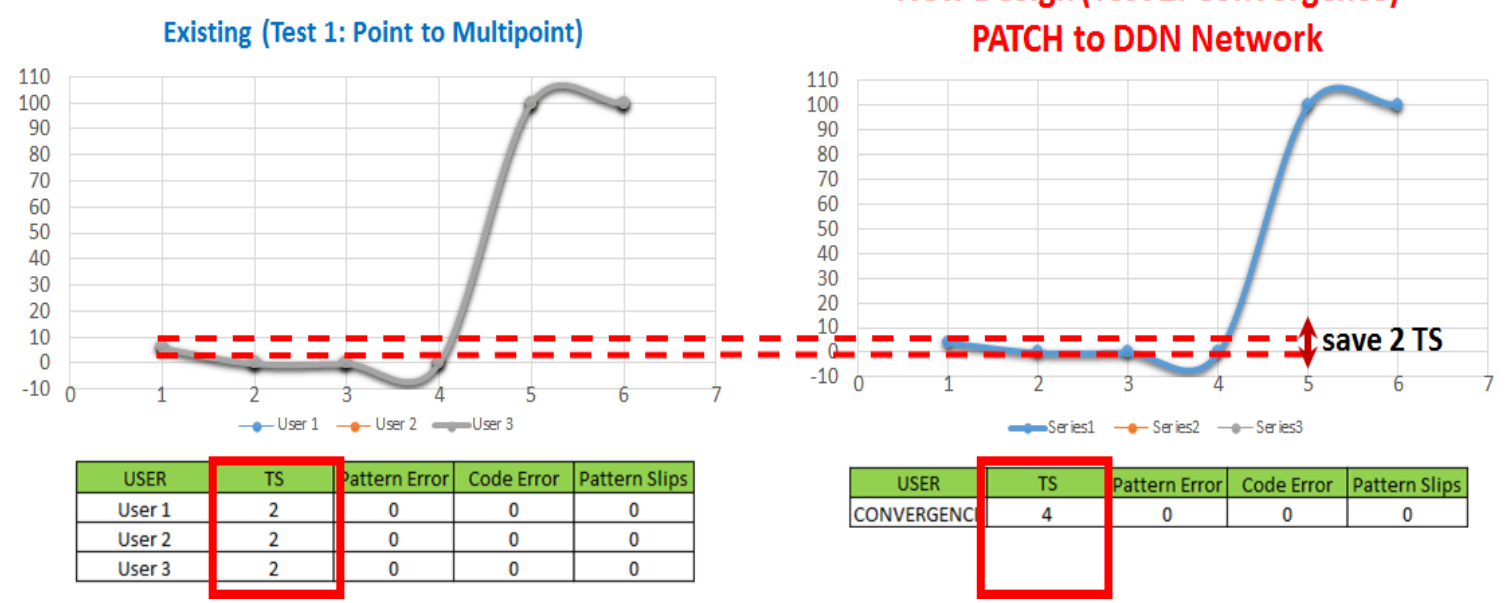

Figure 10. Result of BERT for network connectivity

\subsection{Analysis for the Error Counting for Network 1, Network 2 and Network 3 Between Existing Network and Convergence Network}

This testing is to monitor and verify if there are any error during the BERT Test for 24 hours in both network design. The result as illustrated in Figure 11 shows that the same pattern for the WAN Convergence Network and existing network design. It indicates that there is no error even though the WAN Convergence Network run the multiple network virtually using the less time slots rather than existing network design.

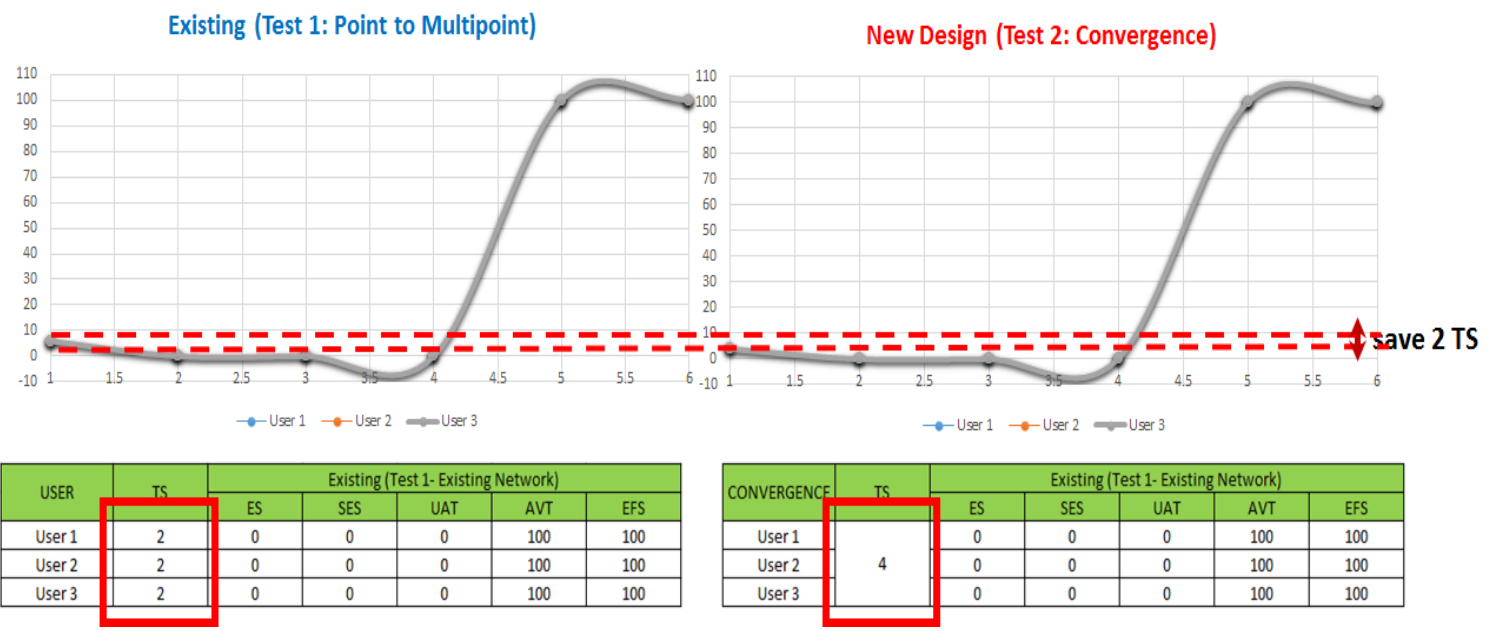

Figure 11. Result of BERT for network quality

\section{CONCLUSION}

This paper presents how the leased line distribution at the EDGE of Local Access Network can be optimized via WAN Convergence Network. The design and implementation of the WAN Convergence Network using a simple concept of SDH structured which channelized the time slots and control the

The optimization of leased line distribution at the edge of local access ... (Nor Shahniza Kamal Bashah) 
transmission line. Through the WAN Convergence Network implementation, it proves that the network infrastructure can be optimized through minimizing the cost of infrastructures development and operation, reducing the risks of service deployment and reducing the operation time to market for new services. The technique used is by relocating the time slots from the multiple leased lines into a WAN Convergence Network and de-multiplexed through the Data Circuit Terminating Equipment (DCE) at the customer premises. Few testing have been conducted between the existing network and the WAN Convergence Network in terms of the transmission medium quality, network connectivity and network quality that proves the WAN Convergence Network is able to support the multiple leased lines through the single line without any error and provide the same quality as the existing network

For further work it is quite relevant to introduce and expand the idea of network convergence to the new technologies such as Metro-E Network and Ethernet in the First Mile (EFM) since all of these technologies are using fully fiber as transmission medium. Since fiber can extend the distance of the network, so these opportunities can help network providers to extend the multiple services to the remote areas.

\section{ACKNOWLEDGEMENTS}

This research is supported by the Research Management Institute, Universiti Teknologi Mara registered under the BESTARI Grant Scheme (FRGS) \#600-IRMI/PERDANA 5/3 BESTARI (098/2018). The authors would like to thank to Research Management Institute, Universiti Teknologi MARA, Malaysia for their funding support and also acknowledged the support given by Crowd Business and Innovation Research Interest Group, Faculty of Computer and Mathematical Sciences, UiTM Shah Alam.

\section{REFERENCES}

[1] Leni Wild et al. "Common Constraints and Incentive Problems in Service Delivery," ODI Working Papers, 2012.

[2] Ben Shenglin et al. "Digital Infrastructure: Overcoming Digital Divide in Emerging Economies," G20 Insights, Jan 15, 2018, [Online] Available: G20 Insights, https://www.g20-insights.org/policy_briefs/digital-infrastructureovercoming-digital-divide-emerging-economies.

[3] Susan Bearden, et al. "Building Technology Infrastructure for Learning Guide," U.S Department of Education, 2017.

[4] Assunção, M.D., Costanzo, A.D., and Buyya, R. "Evaluating the cost-benefit of using cloud computing to extend the capacity of clusters," HPDC, 2009.

[5] Ghezzi, Antonio et al. "A qualitative analysis on the impacts of assured quality internet interconnection," $P E$ WASUN. 2013.

[6] Pin-Han Ho and H. T. Mouftah, "Reconfiguration of spare capacity for MPLS-based recovery in the Internet backbone networks," in IEEE/ACM Transactions on Networking, vol. 12, no. 1, pp. 73-84, Feb. 2004.

[7] Krug, Louise et al. "Understanding the environmental costs of fixed line networking," $e$-Energy, 2014.

[8] Turner, Daniel et al. "California fault lines: understanding the causes and impact of network failures," SIGCOMM, 2010.

[9] Sutherland, Ewan, "The Regulation of Leased Lines," SSRN, Jun 14, 2007, [Online] Available: at SSRN https://ssrn.com/abstract=1752492.

[10] John Chamberlain, "3 Necessities for Network Convergence in 2018 and Beyond," ECNMAG, Jan 15, 2019, [Online] Available: Ecnmag, https://www.ecnmag.com/blog/2018/01/3-necessities-network-convergence-2018-and-beyond.

[11] Christy Roland, "Network Convergence 101: Examples and Perspectives that Form a Connected World," AT\&T SHAPE, Apr 30, 2018, [Online] Available: at AT\&T SHAPE, https://shape.att.com/blog/network-convergence.

[12] Shaikh Abdul Azeem, Satyendra Kumar Sharma, "Study of Types and Practice of Network Convergence," in International Journal on Recent and Innovation Trends in Computing and Communication, vol. 5, no. 5, pp. 696-699, May. 2017.

[13] Ms Jyoti, "Data Communication Network," Biyani’s Think Tank Concept Based Notes, Rajasthan: Think Tanks Biyani Group of Colleges, 2012.

[14] Barnikas, R, Srivasta, J.D, "Power and Communications Cables: Theory and Applications,” IEEE Press, 2003.

[15] Priscilla Oppenheimer, "Characterizing the Existing Internetwork," Top Down Network Design, Third Edition, USA: Cisco Press, 2011.

[16] John Josephakis, “DataDirect Networks Update,” DataDirectTM Networks Information in Motion, Mar. 2013.

[17] Cisco Networking Academy, "Exploring the Modern Computer Network: Types, Functions and Hardware," Network Basics Companion Guide, USA: Cisco Press, Dec 19, 2013.

[18] Center for Digital Government, "Network Convergence: The Right Path for Modernization," CenturyLink® Government Whitepaper, 2016.

[19] Marwan Al-Shawi, "Campus Network Design Models," Network Computing, Aug 23, 2016, [Online] Available: Cisco Press, https://www.networkcomputing.com/data-centers/campus-network-design-models.

[20] Preeta M. Baneerje, “A Network of Networks: How will Carriers Handle the Evolution to 5G,” Deloitte, June 22, 2017, [Online] Available: Deloitte Insights, https://www2.deloitte.com/insights/us/en/topics/emergingtechnologies/5g-next-gen-network-of-networks.html. 
[21] Matin May, "Enterprise Networking: Everything You Need to Know," Extreme Networks, 2014.

[22] O. O. Ayokunle, "Integrating Voice over Internet Protocol (VoIP) Technology as a Communication Tool on a Converged Network in Nigeria," International Journal of Information and Communication Technology Research, vol. 2, no. 11, Nov. 2012.

[23] M. Zanderigo, "The benefits of converged network and application performance management," Nov 15, 2014, [Online] Available at http://www.networkwor.com/article/2452655/application-performance-management/thebenefits-of-converged-network-and-application-performance.html.

[24] Megger Guide, "The Complete Guide to Electrical Insulation Testing," pp. 7-10, 14 Apr, 2014.

[25] RFC 3430, "Simple Network Management Protocol (SNMP) over Transmission Control Protocol (TCP) Transport Mapping," May 9, 2014, [Online] Available at http://www.ietf.org/rfc/rfc3430.txt

\section{BIOGRAPHIES OF AUTHORS}
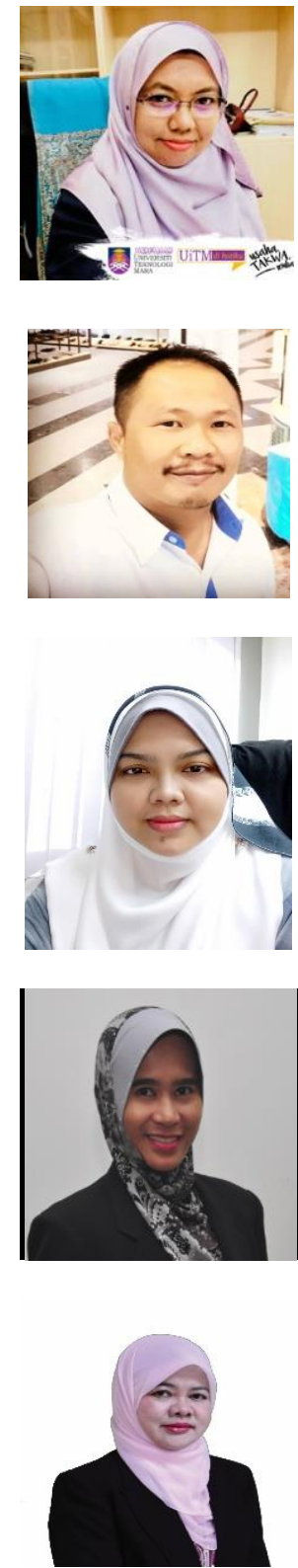

Nor Shahniza Kamal Bashah (Dr.) is a senior lecturer at the Department of Computer and Mathematical Sciences and currently attached as Head of Policy and Strategic ICT at Infostructure Office, Universiti Teknologi MARA, Malaysia. She obtained her PhD in Telematics from Norwegian University of Science and Technology (NTNU), Norway and MSc. in Computer Science major in Distributed Computing from Universiti Putra Malaysia. Her Bachelor Degree was in Computer Science major in Software Engineering from Universit Teknologi Malaysia and her research interest involved in the field of Mobile and Wireless Communication and Semantic Web.

Nor Haizon Husin was a postgraduate student in Computer Networking from Universiti Teknologi, MARA Shah Alam. He is working as an Assistant Manager at Telekom (M) Bhd, Putrajaya. He has wide experiences in the network design and implementation and interested to explore more on emerging and convergence network infrastructure.

Syaripah Ruzaini Syed Aris is a senior lecturer at Faculty of Computer and Mathematical Sciences. She was the Coordinator of Center of Information System Studies, Faculty of Computer and Mathematical Sciences and Head of Post Graduate Study Centre at Centre of Media and Information Warfare, Faculty of Communication and Media Studies, Universiti Teknologi MARA. She obtained her PhD in the field of IT Risk Management. Research interest include IT Management, Risk Management, Information System Strategic Planning and Crowdsourcing. She provides consultation services related to Information System Strategic Planning and Crowdsourcing. She also enjoys giving training to area related to crowdsourcing.

Norjansalika is a senior lecturer in the Faculty of Computer and Mathematical Sciences, Universiti Teknologi MARA, Shah Alam. She is an active researcher and a member of Crowd Business and Innovation Research Interest Group in the University. Her research focus is centered on the broad area of information systems implementation from a socio-technical perspective with particular interest in low income community, organizational context and Islamic contexts.

Noor Habibah Arshad is a Professor at the Faculty of Computer and Mathematical Sciences, Universiti Teknologi MARA (UiTM) and currently assigned as the Assistant Vice Chancellor (Infostructure), UiTM. She is responsible for planning and implementing the University's ICT requirements of all the 35 campuses throughout Malaysia. Noor Habibah Arshad graduated with BSc. in Computer Science from Western Michigan University, MSc. in Management Information Systems from United States International University (San Diego) and a Ph.D in Information Systems from Universiti Kebangsaan Malaysia. 